\title{
Malapposed Struts with Cre8, Biomatrix, and Xience Stents Assessed with OCT Immediately after Implantation and at 6-Month Follow-Up: Can the Different Biomechanical Characteristics of the Three Stents Impact on Struts Malapposition?
}

\author{
Cristina Giglioli $\mathbb{D}^{1,2}$ Emanuele Cecchi, ${ }^{1}$ Chiara Formentini, ${ }^{3}$ Marco Chiostri, ${ }^{3}$ \\ Niccolò Marchionni, ${ }^{1,3}$ and Salvatore Mario Romano ${ }^{1,3}$ \\ ${ }^{1}$ Division of General Cardiology, Azienda Ospedaliero-Universitaria Careggi, Florence, Italy \\ ${ }^{2}$ Department of Cardiovascular and Thoracic Medicine, Azienda Ospedaliero-Universitaria Careggi, Viale G.B. Morgagni 85, \\ Florence 50134, Italy \\ ${ }^{3}$ Department of Experimental and Clinical Medicine, University of Florence, Florence, Italy
}

Correspondence should be addressed to Cristina Giglioli; cristinagiglioli@yahoo.it

Received 17 December 2020; Revised 11 March 2021; Accepted 31 March 2021; Published 10 April 2021

Academic Editor: Leonardo De Luca

Copyright (c) 2021 Cristina Giglioli et al. This is an open access article distributed under the Creative Commons Attribution License, which permits unrestricted use, distribution, and reproduction in any medium, provided the original work is properly cited.

\begin{abstract}
Background. Although the clinical effects of stent malapposition remain controversial, several analyses of stent registries consistently have found that malapposed struts were frequently identified in patients who experienced stent thrombosis. In this study, which is a subanalysis of the previously published CREBX-OCT study, we compared optical coherence tomography (OCT) analysis at the index percutaneous coronary intervention (PCI) and at six-month follow-up in 37 patients randomly assigned to receive, by a single operator, three different second-generation drug-eluting stents (Cre8, Biomatrix, and Xience) aiming to clarify if the malapposition observed at six-month follow-up was persistent or late-acquired. Moreover, we investigated if there were some differences in the behavior of the three different kinds of stents in relation to the struts malapposition. Material and Methods. We analyzed 614 and 599 cross sections and 5514 and 5377 struts at the index PCI and at six-month follow-up, respectively. The qualitative analysis of the plaque composition among the three groups did not show significant differences. Results. The lumen area did not significantly change from the index procedure to the six-month follow-up in the three groups; on the contrary, the number of malapposed struts increased significantly in the Cre8 and Biomatrix groups but not in the Xience group: $0.58 \pm 1.51$ and $3.29 \pm 5.33(p<0.023)$ in the Cre8 group, $0.55 \pm 1.81$ and $1.73 \pm 2.28(p<0.024)$ in the Biomatrix group, and $0.55 \pm 1.5$ and $0.25 \pm 0.87(p<0.166)$ in the Xience group, respectively. Conclusions. Therefore, the malapposition observed at six-month followup in our study population could be mainly considered as acquired and attributable to biomechanical reasons due to the structural differences among the three stents. This trial is registered with Clinical Trials.gov Identifier: NCT02850497.
\end{abstract}

\section{Introduction}

Optical coherence tomography (OCT) allows for high-resolution intracoronary imaging and has been validated for assessment of stent struts coverage and apposition with an accuracy resembling that of histological examination [1-3].
Using OCT to guide stent implantation, it has been found that stent deployment can be associated with stent malapposition to the vessel wall which is too subtle to be detected by angiography or even by intravascular ultrasound. Although the clinical effects of stent malapposition remain controversial, several analyses of stent registries have 
consistently found that malapposed struts were frequently identified in patients who experienced stent thrombosis [4-8]. In the CREBX-OCT study [9], using OCT 6 months after stent implantation, we compared the number of uncovered, protruding, or malapposed struts among the three different second-generation drug-eluting stents (DES) in 60 patients submitted to PCI and randomly assigned to receive Cre8, Biomatrix or Xience. Our previous study showed that the three second-generation stents were similarly effective in permitting complete struts coating 6 months after implantation, but Cre8 and Biomatrix showed a greater proportion of protruding and malapposed struts. These differences did not translate into different risks of MACE over the 6-month follow-up but the study was not powered for detecting differences in the clinical outcome. Although OCT was not mandatory at the time of the index procedure, in 51/60 patients enrolled in the CREBX-OCT study, it was also performed immediately after stent implantation and, in 37 patients, the data recorded were technically suitable for comparative analysis. In this present study, we compared OCT data recorded at the index PCI with those recorded at six-month follow-up in this group of 37 patients. The aim of the study was to clarify whether the malapposition observed at six-month follow-up had persisted since the index PCI or was late-acquired. Moreover, we analyzed any differences in the behavior of the three different kinds of stents in relation to the struts malapposition at the index PCI and at 6-month follow-up.

\section{Methods}

The present study is a subanalysis of the data recorded in patients enrolled in the previously published CREBX-OCT study [9] in a nonprofit, single-center, prospective randomized study (Clinical Trials.gov identifier: NCT02850497). Funding for this subanalysis was provided by Abbott CV, which had no role in the data collection, management, analysis, or results of this study.

2.1. Endpoints. The primary endpoint was to verify if the malapposed struts observed at 6-month follow-up by OCT were late-acquired or were persistent since being recorded by OCT at the index procedure. The coprimary endpoint was to analyze any differences among the three kinds of stents in relation to the struts' malapposition at the index PCI and at 6-month follow-up.

2.2. Patients. Inclusion and exclusion criteria in the study, randomization method to Cre8, Biomatrix, and Xience implantation, stenting, and OCT technique of our study population were previously published in the CREBX-OCT study [9]. Briefly, patients above 18 years of age planned to be treated with PCI for stable angina (SA) or non-ST segment elevation acute coronary syndrome (N-STE ACS) at the catheterization laboratory of the University of Florence from September 2015 to July 2017 were considered eligible for the study. Exclusion criteria were the following: ST segment elevation myocardial infarction, contraindication to dual antiplatelet therapy, surgical intervention planned $<6$ months, indication to anticoagulant therapy, expected survival $<6$ months, cardiogenic shock, unwillingness to sign informed consent or to undergo 6-month coronary angiography.

Randomization to Cre8, Biomatrix, or Xience was performed in computer-generated sequences after coronary angiography resulting in indication to DES implantation. In case of multivessel coronary disease, only one vessel (vessel reference diameter $\geq 25 \mathrm{~mm}$ and without stents previously implanted) was chosen for the study. The study population included 37 out of 60 patients enrolled in the CREBX-OCT study in whom OCT analysis was performed not only at 6month follow-up but also at the index PCI, immediately after stent implantation at operator's discretion. Figure 1 shows flow chart of patients enrolled in the study.

28 patients $(75 \%)$ were males; $14(37 \%)$ were randomized to receive Cre8, $11(29 \%)$ were randomized to receive Biomatrix, and 12 (32\%) were randomized to receive Xience.

2.3. Stents and OCT Analysis. The three types of stents implanted were structurally different.

The polymer-free Cre8 ${ }^{\mathrm{TM}}$ (CID S.p.A, Saluggia, Italy) has a cobalt-chromium platform, coated with "Carbofilm," eluting Amphilimus ${ }^{\mathrm{TM}}$ which is composed of Sirolimus and a mixture of long chain fatty acid, contained in grooves on the stent's outer surface (strut thickness: $80 \mu \mathrm{m}$ ).

The Biomatrix Flex ${ }^{\mathrm{TM}}$ (Biosensor Europe SA, Morges, Switzerland) has a steel platform, eluting Biolimus A9 ${ }^{\mathrm{TM}}$ which is a semisynthetic Sirolimus derivate with improved pharmacokinetic properties. Its poly-lactic acid polymer is biodegradable: the drug and the polymer are present only on the abluminal surface (strut thickness: $112 \mu \mathrm{m}$, polymer thickness: $11 \mu \mathrm{m}$ ).

The Xience ${ }^{\mathrm{TM}}$ V (Abbott Vascular, Santa Clara, California, USA) has a cobalt-chromium platform, eluting Everolimus, with a nonbiodegradable polymer: the drug and the polymer are present on intraluminal as well as on the abluminal stent's surface (strut thickness: $81 \mu \mathrm{m}$; polymer thickness: $3,9 \mu \mathrm{m} \times 2$ ).

In all three arms, DES were deployed by a single operator (C.G.) at a pressure selected at its discretion according to the characteristics of the lesion, and eventual optimization with noncompliant balloon was based on qualitative assessment of postdeployment angiographic images.

OCT was performed with ILLUMIENTM PCI Optimization System-St. Jude Medical (resolution power $20 \mu \mathrm{m}$ ) after completion of index PCI and at 6-month follow-up coronary angiography as previously described [9]. The offline analysis of OCT data was performed by ILLUMIENTM OPTISTM St. Jude Medical workstation by two different investigators (C.F. and E.C.) who were blinded to each other's assessment and to the type of implanted stent as previously described [9]. To obtain identical analysis of segments at baseline and at follow-up OCT, we displayed the baseline and follow-up images side by side and performed serial OCT analysis using information about the motorized pullback speed and landmarks as the presence of calcium 


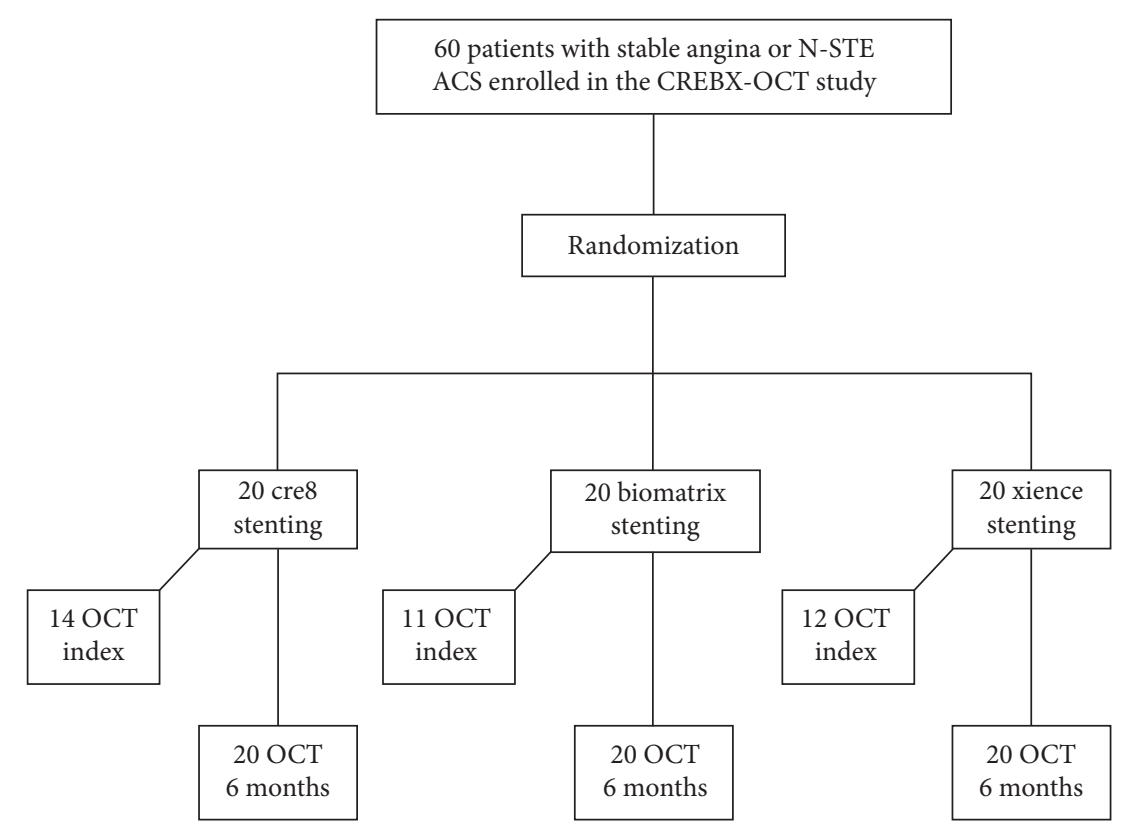

FIGURE 1: Study's flow chart. PCI: percutaneous coronary intervention; N-STE ACS: non-ST segment elevation acute coronary syndrome; OCT: optical coherence tomography.

deposits, side branches, and plaque shape [10]. For qualitative analysis, we evaluated the presence of stent malapposition, thrombus, tissue prolapsed and stent edge dissection, homogeneous struts distribution, and plaque composition.

Struts were classified as apposed or malapposed in relation to their adherence to the vessel wall $[11,12]$. The thickness of struts was different in the three types of stents in relation to the different structural characteristics and to the polymer presence: malapposition was determined by adding the actual strut thickness and polymer thickness to OCT resolution limit as mentioned above. On the basis of this definition, the cutoff to consider a strut as malapposed is different in the three types of stents.

Malapposition identified at the index PCI but absent at follow-up was defined as resolved; otherwise, it was classified as persistent. Late-acquired malapposition was defined as that not present immediately after the index PCI but observed at the follow-up. Tissue prolapsed was defined as a protrusion of tissue between stent struts, extending inside a circular arc connecting adjacent struts. Stent edge dissection was defined as disruption of the luminal vessel surface in the edge segment [10]. Representative serial OCT images of these figures are shown in Figure 2.

2.4. Pharmacological Treatment. Patients treated for SA received a loading dose of $600 \mathrm{mg}$ of Clopidogrel before PCI and patients treated for NST-ACS received a loading dose of $180 \mathrm{mg}$ of Ticagrelor or $60 \mathrm{mg}$ of Prasugrel. All patients received $325 \mathrm{mg}$ of ASA orally or $250 \mathrm{mg}$ intravenously before the procedure and heparin was given as an initial bolus of $70 \mathrm{UI} / \mathrm{kg}$. After the procedure, all patients were treated with ASA $100 \mathrm{mg}$ indefinitely and with Clopidogrel $75 \mathrm{mg}$ daily for six months in patients treated for SA or with
Ticagrelor $180 \mathrm{mg}$ daily or Prasugrel $10 \mathrm{mg}$ daily for almost 12 months in patients with NST-ACS. 34/37 (92\%) patients were taking statins $(12,11$, and 11 patients, respectively in Cre8, Biomatrix, and Xience groups). Other drugs such as beta-blockers and angiotensin-converting enzyme inhibitors were used in accordance with international guidelines. Response to the dual antiplatelet therapy was evaluated by light transmittance aggregometry (LTA) using $10 \mu \mathrm{M} / \mathrm{L}$ adenosine-diphosphate (ADP) and $1 \mathrm{mM}$ arachidonic acid (AA) as agonists. Patients with high on-treatment platelet reactivity (HPR) by $\mathrm{ADP} \geq 70 \%$ were switched to another P2Y12 antagonist [13].

2.5. Statistical Analysis. The statistical software was SPSS (version 20.0; SPSS Inc., Chicago, IL). Normally distributed data were expressed as mean $\pm \mathrm{SD}$, nonnormally distributed data were expressed as median $\left(25^{\text {th }}-75^{\text {th }}\right.$ percentile, i.e., interquartile range, IQR), and categorical data were presented as the frequency (percentage). Sometimes, to better highlight differences, values were reported as both means \pm SD and medians (IQR). Comparisons of variables across the 3 groups were made using one-way analysis of variance (ANOVA) for normal distribution variables, Kruskal-Wallis test for nonnormal distribution variables, and Chi-square test for categorical variables. For intragroup comparisons (i.e., values at follow-up versus index), the parametric paired-samples $t$-test and the nonparametric Wilcoxon test were used. All tests were two-sided. A $p$ value $<0.05$ was considered statistically significant.

\section{Results}

The main baseline demographic, clinical, and angiographic characteristics of the 37 patients included in the present 


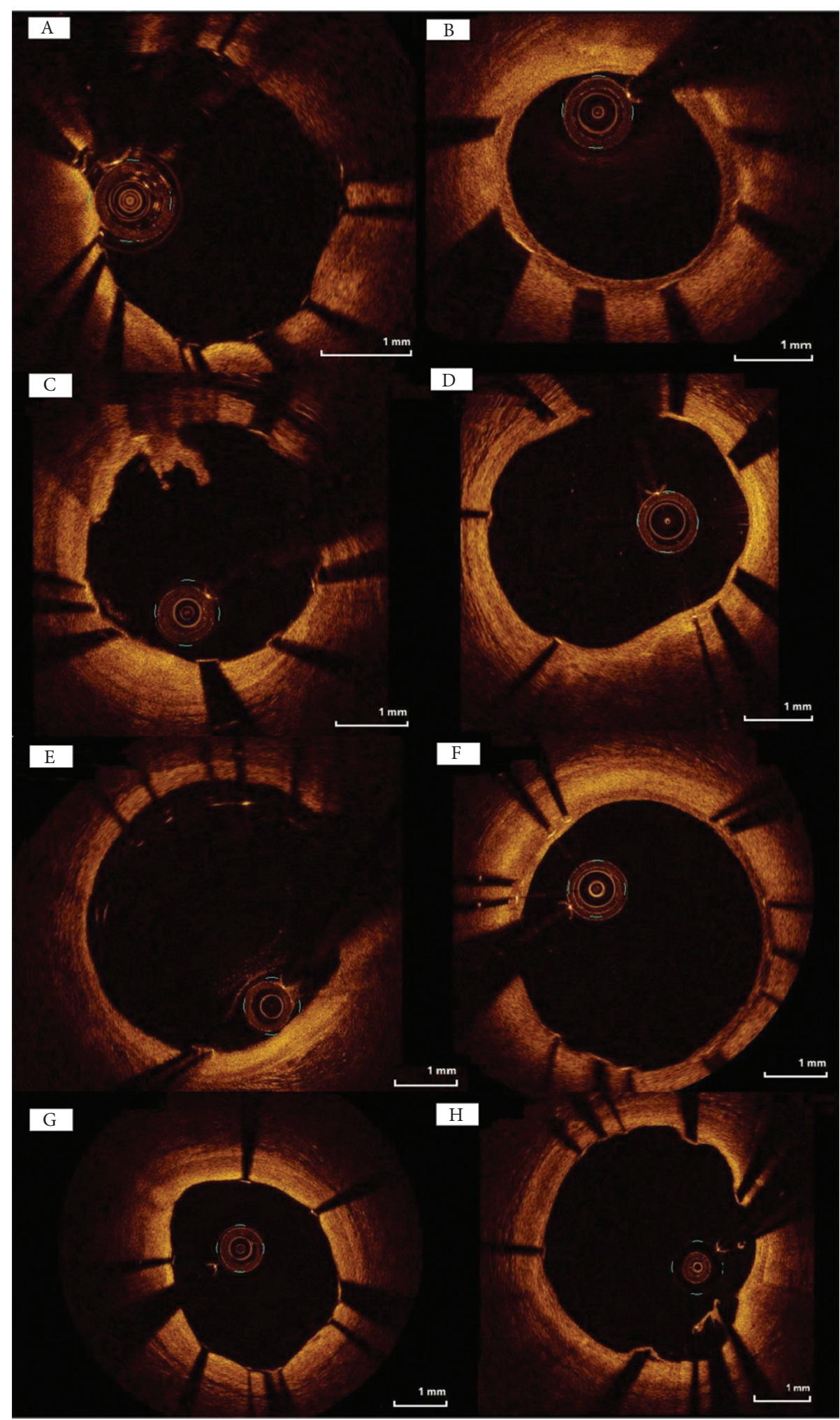

Figure 2: Examples of optical coherence tomography (OCT) cross sections obtained from our study's population: (a) OCT at index percutaneous coronary intervention (PCI); (b) OCT at follow-up. (A) Optimal stent apposition; (B) normal healing; (C) optimal stent apposition with plaque prolapse; (D) normal healing; (E) stent malapposition with incomplete stent apposition area; (F) resolved malapposition; $(\mathrm{G})$ optimal stent apposition; $(\mathrm{H})$ acquired stent malapposition with incomplete stent apposition area. 
study were all similar across the three randomization groups (Table 1). The main laboratory tests at baseline were also similar among the three groups (Table 2). Also, the prevalence of responder patients to ASA and P2Y12 among the three groups did not show any differences in the three groups: ASA responder patients were 13, 10, and 11; P2Y12 responder patients were 11,9 , and 10, respectively, in Cre8, Biomatrix, and Xience groups.

Table 3 shows OCT data recorded at the index procedure and the comparison with data recorded at 6-month followup. The qualitative analysis of the plaque composition among the three groups did not show significant differences and a homogeneous distribution of the struts was present in all groups. The lumen area had not significantly changed in the period between the index procedure and the 6-month follow-up in the three groups. On the contrary, the number of malapposed struts increased significantly in the Cre8 and Biomatrix groups but not in the Xience group. Similarly, the number of cross sections with incomplete stent apposition (ISA) increased significantly in the Cre8 and Biomatrix groups but not in the Xience group.

\section{Discussion}

The main findings of our analysis were the following:

(1) The number of malapposed struts increased significantly from the index procedure to the six-month follow-up. Therefore, we can state that the malapposition observed by OCT at six-month follow-up was mainly late-acquired.

(2) The three different kinds of DES showed a different behavior in relation to the struts' malapposition during the follow-up: despite a similar number of malapposed struts at the index PCI, the number of malapposed struts had increased in the Cre8 and Biomatrix groups but not in the Xience group at sixmonth follow-up. We hypothesized that these differences among the three groups could be ascribed to biomechanical reasons due to the different structure of the stents.

These findings deserve some consideration.

The intracoronary imaging techniques indicate that struts' malapposition is a relatively frequent phenomenon after stent implantation, observed in a portion of stents analyzed ranging from $25 \%$ to $60 \%$ [10]. There are three types of stent malapposition: acute, late-persistent, and lateacquired. Acute struts' malapposition may result from incomplete stent expansion during the index procedure, especially in the presence of calcified plaques. Late malapposition may be persistent from the index PCI or acquired due to either the presence of thrombus or dissection between stent and plaque at stent implantation, which may have disappeared at follow-up, or due to positive vessel remodeling $[14,15]$. In the era of drug-eluting stents, late-acquired stent malapposition is a well-recognized problem in interventional cardiology because it may constitute a potent substrate for late stent thrombosis. The clinical role of late malapposition is somewhat controversial: several experimental and clinical studies suggested that stent malapposition has a direct impact on thrombus formation, and it is frequently identified in patients who experienced stent thrombosis [16-18]. Conversely, some long-term follow-up studies by OCT showed a favorable clinical outcome for patients with stent malapposition [4-8]. In our study's population, the number of struts malapposed and cross sections with ISA increased significantly from baseline OCT to 6-month follow-up in the Cre8 and Biomatrix groups but not in the Xience group. Although these differences did not translate into different risks of MACE over the 6-month follow-up because the study was not powered to detect differences in the clinical outcome, it could be useful for interventional cardiologists to be aware of the different behavior of the three types of stents so that they can choose the safest and most suitable type for their patients. Moreover, it could be interesting to speculate about the reasons for these differences. First of all, we can reasonably exclude the fact that the different results among the three groups were ascribable to differences in patients' characteristics (Tables 1-3) or in interventional techniques (all PCI were performed by a single operator); therefore we can suppose that the differences observed were mainly of biomechanical nature (Figure 3).

As specified above, the three types of stents are coated with three different biodegradable drugs with possible different biochemical effects on the vessel wall. Moreover, they differ from one another according to the presence and the type (biodegradable or not) of the polymer interposed between the metal platform and the vessel wall as well as the shape of the polymer containers on the platform surface. The Biomatrix stent, which has a biodegradable polymer on the abluminal surface of the platform, even though well-apposed to the vessel wall at the index PCI, at six-month follow-up may show an empty space between the platform and the vessel wall due to drug and polymer reabsorption. This empty space may be larger than that observed at six-month follow-up in the Xience stent, in which only the drug is reabsorbed, as the polymer is not biodegradable. Otherwise, Cre8 stents have no polymer on the platform surface and after the reabsorption of the drug they could show an empty space not larger but more heterogeneous than that of the other two stents, due to their structure with grooves on the platform surface. To sum up, at six-month follow-up, the Xience stent may show an empty space between platform and vessel wall, which is smaller than that observed in the Biomatrix stent and more homogeneous than that observed in the Cre8 stent. Therefore, the structural characteristics of the Xience stent could allow better contact with and greater homogeneous adherence to the vessel wall when compared to the other two stents. The differences in the dimensions and in the homogeneity of the empty space between the platform and the vessel wall may also be associated with differences in the shear pressure and flow disturbance between stent and vessel wall able to determine differential changes in cellular proliferation of the vessel wall among the three groups. It is known that a high shear flow disturbance is a factor of delay of neointimal growth, which may lead to a permanence of malapposition [19-21]. 
TABLE 1: Baseline clinical, angiographic, and PCI characteristics.

\begin{tabular}{|c|c|c|c|c|}
\hline & Cre8 & Biomatrix & Xience & $p$ value \\
\hline Patients $(n)$ & 14 & 11 & 12 & \\
\hline Gender M/F, $n(\%)$ & $11 / 3(78.6 / 21.4)$ & $7 / 4(63.6 / 36.4)$ & $10 / 2(83.3 / 16.7)$ & 0.519 \\
\hline Italian, $n(\%)$ & $14(100)$ & $11(100)$ & $11(91.7)$ & 0.343 \\
\hline Age (years), mean $\pm S D$ & $66.6 \pm 10.0$ & $67.1 \pm 11.1$ & $57.9 \pm 12.2$ & 0.087 \\
\hline $\mathrm{BMI}\left(\mathrm{Kg} / \mathrm{m}^{2}\right)$, mean $\pm \mathrm{SD}$ & $26.9 \pm 2.9$ & $24.9 \pm 1.6$ & 28.23 .0 & 0.036 \\
\hline Hypertension, $n(\%)$ & $12(85.7)$ & $10(90.9)$ & $11(91.7)$ & 0.867 \\
\hline Diabetes, $n(\%)$ & $4(28.6)$ & $3(27.3)$ & $3(25)$ & 0.979 \\
\hline Dyslipidemia, $n(\%)$ & $11(78.6)$ & $9(81.8)$ & $9(75)$ & 0.924 \\
\hline Ever smoker, $n(\%)$ & $11(78.6)$ & $5(45.5)$ & $7(58.3)$ & 0.225 \\
\hline Familial history of CAD, $n(\%)$ & $4(28.6)$ & $5(45.5)$ & $2(16.7)$ & 0.318 \\
\hline Previous CAD, $n(\%)$ & $4(28.6)$ & $1(9.1)$ & $1(8.3)$ & 0.282 \\
\hline Previous PCI, $n(\%)$ & $2(14.3)$ & $0(0.0)$ & $0(0.0)$ & 0.176 \\
\hline Previous CABG, $n(\%)$ & $0(0.0)$ & $0(0.0)$ & $0(0.0)$ & - \\
\hline Previous TIA/stroke, $n(\%)$ & $1(7.1)$ & $0(0.0)$ & $0(0.0)$ & 0.430 \\
\hline Chronic kidney disease, $n(\%)$ & $1(7.1)$ & $0(0.0)$ & $0(0.0)$ & 0.430 \\
\hline COPD, $n(\%)$ & $1(7.1)$ & $2(18.2)$ & $0(0.0)$ & 0.276 \\
\hline Sinus rhythm, $n(\%)$ & $11(100.0)$ & $9(81.8)$ & $11(91.7)$ & 0.255 \\
\hline Atrial fibrillation, $n(\%)$ & $0(0.0)$ & $1(9.1)$ & $0(0.0)$ & 0.297 \\
\hline Pacemaker, $n(\%)$ & $0(0.0)$ & $1(9.1)$ & $1(8.3)$ & 0.524 \\
\hline LVEF, mean \pm SD $(\%)$ & $57 \pm 6.4$ & $59 \pm 7.3$ & $53 \pm 8.9$ & 0.164 \\
\hline Heart failure, $n(\%)$ & $0(0.0)$ & $1(9.1)$ & $1(8.3)$ & 0.524 \\
\hline NYHA class & & & & 0.598 \\
\hline $\mathrm{I}^{\circ}, n(\%)$ & $13(92.9)$ & $9(81.8)$ & $10(83.3)$ & \\
\hline $\mathrm{II}^{\circ}, n(\%)$ & $0(0.0)$ & $0(0.0)$ & $1(8.3)$ & \\
\hline $\mathrm{III}^{\circ}, n(\%)$ & $1(7.1)$ & $1(9.1)$ & $1(8.3)$ & \\
\hline $\mathrm{IV}^{\circ}, n(\%)$ & $0(0.0)$ & $1(9.1)$ & $0(0.0)$ & \\
\hline Stable angina, $n(\%)$ & $2(14.3)$ & $3(27.3)$ & $3(25.0)$ & 0.693 \\
\hline Unstable angina, $n(\%)$ & $6(42.9)$ & $5(45.5)$ & $2(16.7)$ & 0.262 \\
\hline Silent ischemia, $n(\%)$ & $1(7.1)$ & $0(0.0)$ & $2(16.7)$ & 0.338 \\
\hline NSTEMI, $n(\%)$ & $3(21.4)$ & $3(27.3)$ & $3(25.0)$ & 0.942 \\
\hline STEMI, other vessels, $n(\%)$ & $3(21.4)$ & $0(0.0)$ & $2(16.7)$ & 0.276 \\
\hline \multicolumn{5}{|l|}{ Target lesion coronary artery } \\
\hline Left anterior descending & $12(85.7)$ & $9(81.8)$ & $11(91.7)$ & 0.784 \\
\hline Left circumflex & $7(50.0)$ & $4(36.4)$ & $7(58.3)$ & 0.570 \\
\hline Right & $8(57.1)$ & $6(54.5)$ & $7(58.3)$ & 0.983 \\
\hline Left main & $0(0.0)$ & $1(9.1)$ & $0(0.0)$ & 0.297 \\
\hline
\end{tabular}

BMI: body mass index, CAD: coronary artery disease, PCI: percutaneous coronary intervention, CABG: coronary artery by-pass graft, TIA: transient ischemic attack, COPD: chronic obstructive pulmonary disease, LVEF: left ventricle ejection fraction.

TABle 2: Baseline biohumoral data.

\begin{tabular}{|c|c|c|c|c|}
\hline & Cre8 & Biomatrix & Xience & $p$ value \\
\hline Pro-BNP (pg/mL), median (IQR) & $661(377-1551)$ & $136(87-804)$ & $242(78-660)$ & 0.286 \\
\hline Troponin $(\mu \mathrm{g} / \mathrm{L})$, median (IQR) & $0.60(0.16-2.01)$ & $1.08(0.41-1.79)$ & $2.06(0.41-5.14)$ & 0.577 \\
\hline Hemoglobin $(\mathrm{g} / \mathrm{dL})$ & $12.3 \pm 2.1$ & $12.4 \pm 1.1$ & $13.2 \pm 1.8$ & 0.427 \\
\hline Leukocyte $(109 / \mathrm{L})$ & $7.90 \pm 2.70$ & $6.50 \pm 2.30$ & $8.00 \pm 1.70$ & 0.233 \\
\hline Platelets $(109 / \mathrm{L})$ & $193 \pm 77$ & $185 \pm 54$ & $201 \pm 52$ & 0.822 \\
\hline Creatinine $(\mathrm{mg} / \mathrm{dL})$ & $0.99 \pm 0.47$ & $0.83 \pm 0.25$ & $0.96 \pm 0.26$ & 0.501 \\
\hline Total cholesterol (mg/dL) & $165 \pm 46$ & $151 \pm 27$ & $188 \pm 68$ & 0.287 \\
\hline HDL cholesterol (mg/dL) & $44 \pm 16$ & $39 \pm 9$ & $40 \pm 17$ & 0.739 \\
\hline LDL cholesterol (mg/dL) & $97 \pm 39$ & $74 \pm 36$ & $113 \pm 53$ & 0.175 \\
\hline Triglycerides (mg/dL) & $100 \pm 54$ & $139 \pm 59$ & $176 \pm 88$ & 0.090 \\
\hline
\end{tabular}

HDL: high density lipoprotein, LDL: low density lipoprotein, pro-BNP: pro-brain natriuretic peptide.

4.1. Clinical Implications. In the choice of stents, interventional cardiologists should consider those structural characteristics, which allow a close and homogeneous contact of the stent with the vessel wall as being an important parameter. In this respect, Xience, among the three types of stents analyzed, would seem to be the best option for daily practice when considering struts' malapposition. 
TABLE 3: OCT analysis at the index PCI and comparison with OCT analysis at 6-month follow-up.

\begin{tabular}{|c|c|c|c|c|c|}
\hline \multicolumn{2}{|c|}{ 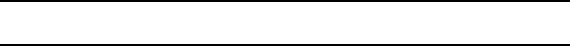 } & Cre8 $(n=14)$ & Biomatrix $(n=11)$ & Xience $(n=12)$ & $p$ value \\
\hline \multicolumn{2}{|c|}{ Stent total length $(\mathrm{mm})$, mean $\pm \mathrm{SD}$} & $20 \pm 7.17$ & $28 \pm 8.92$ & $30.3 \pm 11.83$ & $0.022(\mathrm{~A})$ \\
\hline Overlapping stents, $n$ (\% & & $3(21)$ & $3(27)$ & $4(33)$ & N.S. \\
\hline \multicolumn{6}{|c|}{ Cross sections analyzed $(n)$} \\
\hline \multicolumn{2}{|l|}{ Index PCI } & 221 & 186 & 207 & $\mathrm{~N} / \mathrm{A}$ \\
\hline \multicolumn{2}{|l|}{ Follow-up } & 217 & 179 & 203 & $\mathrm{~N} / \mathrm{A}$ \\
\hline \multicolumn{2}{|c|}{ Homogeneous distribution struts, $n(\%)$} & $98.7 \pm 3.3$ & $97.5 \pm 4.3$ & $99.5 \pm 1.6$ & $0.348(\mathrm{~A})$ \\
\hline \multicolumn{2}{|c|}{ Vessel wall injury, median (IQR) } & $0.0(0.0-0.0)$ & $0.0(0.0-0.0)$ & $0.0(0.0-5.4)$ & $0.010(\mathrm{~K}-\mathrm{W})$ \\
\hline \multicolumn{2}{|c|}{$\begin{array}{l}\text { Vessel lumen without thrombus, } \\
\text { mean } \pm S D\end{array}$} & $97.9 \pm 4.1$ & $96.8 \pm 4.3$ & $96.2 \pm 5.5$ & $0.667(\mathrm{~A})$ \\
\hline \multicolumn{2}{|c|}{ Protruding plaque, median (IQR) } & $9.1(0.0-22.2)$ & $0.0(0.0-0.0)$ & $10.5(2.8-16.2)$ & $0.097(\mathrm{~K}-\mathrm{W})$ \\
\hline \multicolumn{2}{|c|}{ Lipid plaque, median (IQR) } & $7.1(0.0-15.8)$ & $0.0(0.0-6.7)$ & $5.9(0.0-13.6)$ & $0.500(\mathrm{~K}-\mathrm{W})$ \\
\hline \multicolumn{2}{|c|}{ Calcified plaque, median (IQR) } & $0.0(0.0-15.4)$ & $0.0(0.0-6.7)$ & $0.0(0.0-14.2)$ & $0.579(\mathrm{~K}-\mathrm{W})$ \\
\hline \multicolumn{2}{|c|}{ Intimal dissection, median (IQR) } & $0.0(0.0-0.0)$ & $0.0(0.0-0.0)$ & $0.0(0.0-0.0)$ & $0.347(\mathrm{~K}-\mathrm{W})$ \\
\hline \multicolumn{6}{|c|}{ Struts analyzed $(n)$} \\
\hline \multicolumn{2}{|l|}{ Index PCI } & 2079 & 1615 & 1820 & $\mathrm{~N} / \mathrm{A}$ \\
\hline \multirow{2}{*}{\multicolumn{2}{|c|}{$\begin{array}{l}\text { Follow-up } \\
\text { Lumen area }\left(\mathrm{mm}^{2}\right), \text { mean }+\mathrm{SD}\end{array}$}} & 2041 & 1554 & 1782 & N/A \\
\hline & & & & & \\
\hline \multicolumn{2}{|l|}{ Index PCI } & $8.70 \pm 2.26$ & $8.66 \pm 2.54$ & $7.15 \pm 1.40$ & 0.138 \\
\hline \multicolumn{2}{|l|}{ Follow-up } & $8.05 \pm 2.38$ & $8.23 \pm 2.87$ & $7.77 \pm 1.25$ & 0.895 \\
\hline \multicolumn{2}{|c|}{ Follow-up versus Index, $p$ (paired $t$-test) } & 0.800 & 0.744 & 0.094 & \\
\hline \multicolumn{6}{|c|}{ Cross section with ISA } \\
\hline \multicolumn{2}{|l|}{ Index PCI, $n(\%)$} & $7 / 220(3.2)$ & 3/186 (1.6) & $7 / 207(3.4)$ & $0.509 \times 2$ \\
\hline \multicolumn{2}{|l|}{ Follow-up, $n(\%)$} & $24 / 217(11.05)$ & $\begin{array}{c}8 / 179(4.5) \\
p \text { value }=0.017 \\
\text { Cre8 versus biomatrix }\end{array}$ & $\begin{array}{c}2 / 203(0.9) \\
p \text { value }=<0.001 \\
\text { Xience versus Cre } 8 \\
p \text { value }=0.033 \\
\text { Xience versus biomatrix }\end{array}$ & $<0.001 \times 2$ \\
\hline \multicolumn{6}{|l|}{ Malapposed struts } \\
\hline Index PCI & $\begin{array}{l}\text { mean } \pm \mathrm{SD} \\
\text { Median (IQR) }\end{array}$ & $\begin{array}{c}0.57 \pm 1.51 \\
0.00(0.00-0.00)\end{array}$ & $\begin{array}{c}0.55 \pm 1.81 \\
0.00(0.00-0.00)\end{array}$ & $\begin{array}{c}0.58 \pm 1.51 \\
0.00(0.00-0.00)\end{array}$ & $\begin{array}{c}0.998(\mathrm{~A}) \\
0.917(\mathrm{~K}-\mathrm{W})\end{array}$ \\
\hline Follow-up & $\begin{array}{l}\text { mean } \pm \text { SD } \\
\text { Median (IQR) }\end{array}$ & $\begin{array}{c}3.29 \pm 5.33 \\
1.00(0.00-4.00) \#\end{array}$ & $\begin{array}{c}1.73 \pm 2.28 \\
1.00(0.00-3.00)\end{array}$ & $\begin{array}{c}0.25 \pm 0.87 \\
0.00(0.00-0.00) \#\end{array}$ & $\begin{array}{c}0.109(\mathrm{~A}) \\
0.032(\mathrm{~K}-\mathrm{W})\end{array}$ \\
\hline Follow-up versus Index & $\begin{array}{l}p \text { (paired } t \text {-test }) \\
p \text { (Wilcoxon) }\end{array}$ & $\begin{array}{l}0.023 \\
0.012\end{array}$ & $\begin{array}{l}0.024 \\
0.026\end{array}$ & $\begin{array}{l}0.166 \\
0.157\end{array}$ & \\
\hline
\end{tabular}

A : ANOVA; $\chi 2$ : chi-square; N/A : not available; K-W: Kruskal-Wallis test. \#: Mann-Whitney $U$-test; $p=0.029$.

Index PCI

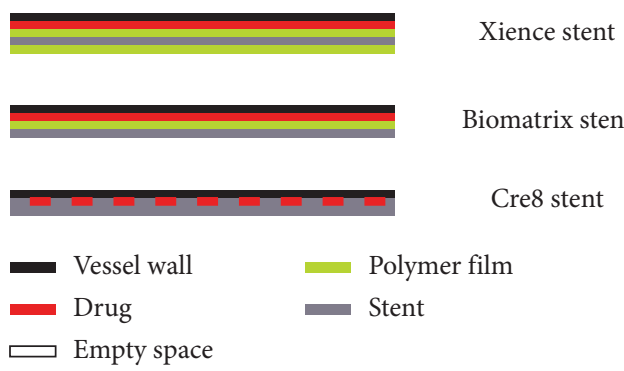

Six-month follow-up

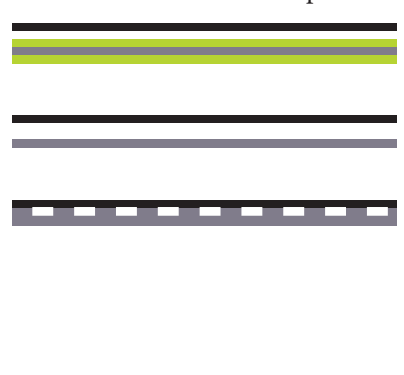

FIGURE 3: Schematic representation of the structural differences among the tree types of stents and their different interaction with the vessel wall during PCI and at 6-month follow-up.

4.2. Limitations. Some limitations of our study have to be acknowledged. First, the study examines only 37 patients and was therefore not powered to allow clinical considerations. Moreover, because of the small number of patients included in this study, the patients in the Xience group were 10 years younger than those in the other two groups. Consequently, we cannot exclude a possible role of aging in the speculative interpretation of the results. The younger patients might have a better long-term healing process compared to older patients.

Second, our conclusions regarding the impact of biomechanical differences on the behavior of the three types of stents are mainly based on logical hypotheses of pathophysiological nature and not on our experimental data. 
4.3. Future Directions. Experimental studies or clinical studies enrolling a wider population of patients will better clarify the relation between stents' structural characteristics and their healing process.

\section{Conclusions}

The results of the present study suggest that the plaque composition and struts' malapposition observed with OCT at the index PCI were not significantly different among the three groups of patients. However, patients treated with Cre8 and Biomatrix showed a higher percentage of malapposed struts than those treated with Xience at six-month follow-up. Therefore, the malapposition observed at six-month followup in our study's population could be mainly considered as acquired and attributable to biomechanical reasons due to the structural differences among the three stents. A close and homogeneous contact of the stents' platform with the vessel wall, as we supposed for the Xience stent, could explain the lower percentage of malapposed struts observed in the group of patients treated with this kind of stent.

\section{Data Availability}

Data supporting our results are collected in a dedicated database of the AOU Careggi Hospital.

\section{Conflicts of Interest}

The authors declare that they have no conflicts of interest.

\section{Acknowledgments}

Funding for this substudy was provided by Abbott CV.

\section{References}

[1] L. Räber, S. Baumgartner, H. M. G. Garcia et al., "Long-term vascular healing in response to sirolimus- and paclitaxeleluting stents," JACC: Cardiovascular Interventions, vol. 5, no. 9, pp. 946-957, 2012.

[2] F. Prati, M. Zimarino, E. Stabile et al., "Does optical coherence tomography identify arterial healing after stenting? An in vivo comparison with histology, in a rabbit carotid model," Heart, vol. 94, no. 2, pp. 217-221, 2008.

[3] A. Murata, D. Wallace-Bradley, A. Tellez et al., "Accuracy of optical coherence tomography in the evaluation of neointimal coverage after stent implantation," JACC: Cardiovascular Imaging, vol. 3, no. 1, pp. 76-84, 2010.

[4] S.-J. Hong, K.-H. Park, C.-M. Ahn et al., "Severe acute stent malapposition follow-up: 3 month and 12 month serial quantitative analyses by optical coherence tomography," International Journal of Cardiology, vol. 299, no. 19, pp. 81-86, 2020.

[5] S. Y. Lee, E. Im, S. J Hong et al., "Severe acute stent malapposition after drug-eluting stent implantation: effects on long-term clinical outcomes," Journal of the American Heart Association, vol. 8, Article ID e012800, 2019.

[6] G. Souteyrand, N. Amabile, L Mangin et al., "Mechanisms of stent thrombosis analysed by optical coherence tomography: insights from the national PESTO French registry," European Heart Journal, vol. 37, pp. 1208-1216, 2016.
[7] M. Taniwaki, M. D. Radu, S. Zaugg et al., "Mechanisms of very late drug-eluting stent thrombosis assessed by optical coherence tomography," Circulation, vol. 133, no. 7, pp. 650-660, 2016.

[8] T. Adriaenssens, M. Joner, T. C. Godschalk et al., "Optical coherence tomography findings in patients with coronary stent thrombosis," Circulation, vol. 136, no. 11, pp. 1007-1021, 2017.

[9] C. Giglioli, C. Formentini, S. M. Romano et al., "Vulnerable struts with CRE8, Biomatrix and Xience stents assessed with OCT and their correlation with clinical variables at 6 month follow-up: the CREBX-OCT study," The International Journal of Cardiovascular Imaging, vol. 36, no. 2, pp. 217-230, 2020.

[10] H. Kawamori, J. Shite, T. Shinke et al., "Natural consequence of post-intervention stent malapposition, thrombus, tissue prolapse, and dissection assessed by optical coherence tomography at mid-term follow-up," European Heart JournalCardiovascular Imaging, vol. 14, no. 9, pp. 865-875, 2013.

[11] F. Prati, E. Regar, G. S. Mintz et al., "Expert review document on methodology, terminology and clinical applications of optical coherence tomography: physical principles, methodology of image acquisition, and clinical application for assessment of coronary arteries and atherosclerosis," European Heart Journal, vol. 31, no. 4, pp. 401-415, 2010.

[12] F. Prati, G. Guagliumi, G. S. Mintz et al., "Expert review document part 2: methodology, terminology and clinical applications of optical coherence tomography for the assessment of interventional procedures," European Heart Journal, vol. 33, no. 20, pp. 2513-2520, 2012.

[13] E. Cecchi, R. Marcucci, M. Chiostri et al., "Dual antiplatelet therapy tailored on platelet function test after coronary stent implantation: a real-world experience," Internal and Emergency Medicine, vol. 10, no. 7, pp. 805-814, 2015.

[14] E. Im, B.-K. Kim, Y.-G. Ko et al., "Incidences, predictors, and clinical outcomes of acute and late stent malapposition detected by optical coherence tomography after drug-eluting stent implantation," Circulation: Cardiovascular Interventions, vol. 7, no. 1, pp. 88-96, 2014.

[15] S. Yamamura, K. Fujisue, K Tsujita et al., "Optical coherence tomography visualization of stent deformation with subsequent thrombus adhesion at very early phase after everolimuseluting stent implantation: a case report," BMC Cardiovascular Disorders, vol. 16, p. 116, 2016.

[16] N. Foin, S. Lu, J. Ng et al., "Stent malapposition and the risk of stent thrombosis: mechanistic insights from an in vitro model," EuroIntervention, vol. 13, no. 9, pp. e1096-e1098, 2017.

[17] H. Mori, M. Joner, A. V. Finn, and R. Virmani, "Malapposition: is it a major cause of stent thrombosis?" European Heart Journal, vol. 37, no. 15, pp. 1217-1219, 2016.

[18] F. Prati, T. Kodama, E. Romagnoli et al., "Suboptimal stent deployment is associated with subacute stent thrombosis: optical coherence tomography insights from a multicenter matched study. From the CLI Foundation investigators: the CLI-THRO study," American Heart Journal, vol. 169, no. 2, pp. 249-256, 2015.

[19] N. Foin, J. L. Gutiérrez-Chico, S. Nakatani et al., "Incomplete stent apposition causes high shear flow disturbances and delay in neointimal coverage as a function of strut to wall detachment distance," Circulation: Cardiovascular Interventions, vol. 7, no. 2, pp. 180-189, 2014.

[20] E. Cecchi, C. Giglioli, S. Valente et al., "Role of hemodynamic shear stress in cardiovascular disease," Atherosclerosis, vol. 214, pp. 249-256, 2010. 
[21] P. Suwannasom, Y. Onuma, E. Benit et al., "Evaluation of vascular healing of polymer-free sirolimus-eluting stents in native coronary artery stenosis: a serial follow-up at three and six months with optical coherence tomography imaging," EuroIntervention, vol. 12, no. 5, pp. e574-e583, 2016. 